\title{
Variational symmetries and pluri-Lagrangian structures for integrable hierarchies of PDEs
}

\author{
Matteo Petrera ${ }^{1}$ - Mats Vermeeren ${ }^{1,2}$
}

Received: 13 June 2019 / Accepted: 1 October 2020 / Published online: 9 November 2020

(c) The Author(s) 2020

\begin{abstract}
We investigate the relation between pluri-Lagrangian hierarchies of 2-dimensional partial differential equations and their variational symmetries. The aim is to generalize to the case of partial differential equations the recent findings in Petrera and Suris (Nonlinear Math. Phys. 24(suppl. 1):121-145, 2017) for ordinary differential equations. We consider hierarchies of 2-dimensional Lagrangian PDEs (many of which have a natural $(1+1)$-dimensional space-time interpretation) and show that if the flow of each PDE is a variational symmetry of all others, then there exists a pluriLagrangian 2-form for the hierarchy. The corresponding multi-time Euler-Lagrange equations coincide with the original system supplied with commuting evolutionary flows induced by the variational symmetries.
\end{abstract}

Keywords Integrable PDEs · Variational principles · Variational symmetries

Mathematics Subject Classification 37K05 · 37K10

\section{Introduction}

In the last decade a variational perspective on integrable systems has emerged under the name of pluri-Lagrangian systems (or Lagrangian multiform systems). The the-

The authors are partly supported by the DFG (Deutsche Forschungsgemeinschaft) in the frame of SFB/TRR 109 "Discretization in Geometry and Dynamics".

$\bowtie \quad$ Mats Vermeeren

vermeeren@math.tu-berlin.de; m.vermeeren@leeds.ac.uk

Matteo Petrera

petrera@math.tu-berlin.de

1 Institut für Mathematik, MA 7-1, Technische Universität Berlin, Str. des 17. Juni 136, 10623

Berlin, Germany

2 Present Address: School of Mathematics, University of Leeds, Leeds LS2 9JT, UK 
ory was initiated in [13] in the discrete setting, more specifically in the context of multidimensionally consistent lattice equations on a quadrilateral stencil, called quad equations. Multidimensional consistency means that the equation can be imposed on all elementary squares in a higher-dimensional lattice without leading to contradictions. Analogously to commutativity of differential equations, multidimensional consistency is a key feature of integrability for difference equations.

In [13] it was shown that the property of multidimensional consistency can be combined with a variational formulation for quad equations. Solutions of integrable quad equations are critical points of an action functional obtained by integrating a suitable discrete Lagrangian 2-form over an arbitrary 2-dimensional surface in a higher-dimensional lattice. If the 2-dimensional surface is a plane, we recover a traditional variational principle for a 2-dimensional difference equation where the action is the sum over a plane of evaluations of the Lagrange function. The pluri-Lagrangian property requires the action to be critical also when this plane is replaced by any other 2-dimensional discrete surface in a higher-dimensional lattice. This remarkable property has been considered as a defining feature of integrability of 2-dimensional discrete equations $[2,4,6,9,13-15,32]$ as well as in the 1-dimensional $[5,7,33]$ and 3-dimensional $[8,16]$ cases.

The pluri-Lagrangian property can also be formulated in the continuous case, where solutions of (hierarchies of) integrable 2-dimensional partial differential equations (PDEs) are critical points of an action functional obtained by integrating a differential 2 -form over an arbitrary 2-dimensional surface in a higher-dimensional space. This variational principle has been proposed as a Lagrangian analogue of the existence of Poisson-commuting Hamilton functions [13,27,28,32]. As in the discrete case, it is not limited to Lagrangian 2-forms describing 2-dimensional PDEs. The corresponding variational principle where a Lagrangian 1-form is integrated over curves applies to integrable ordinary differential equations [22,26,33]. It is conjectured that also for $d>2$ integrable hierarchies of $d$-dimensional integrable PDEs can be described by pluri-Lagrangian $d$-forms.

Thanks to these investigations a quite suggestive scenario has emerged: the pluriLagrangian structure is closely related (or even equivalent) to the integrability of the underlying system. This novel characterization of integrability applies to both ordinary differential (or difference) equations and partial differential (or difference) equations.

In the recent paper [22] a connection between the notions of pluri-Lagrangian structures and variational symmetries was proved in the context of classical mechanics. In particular, it was shown that the existence of commuting variational symmetries for a system of variational ordinary differential equations leads to a natural pluri-Lagrangian 1-form, whose multi-time Euler-Lagrange equations consist of the original system and commuting flows corresponding to the variational symmetries. These findings confirmed, in the framework of classical mechanics, that a pluri-Lagrangian structure is hidden behind the existence of a sufficient number of variational symmetries (i.e., of integrals of motion thanks to Noether theorem).

In the present work we extend the above idea to the case of variational 2-dimensional PDEs, thus generalizing the results of [22] to the context of Lagrangian field theory with two independent variables. We consider hierarchies of variational PDEs where the flow of each PDE is a variational symmetry of the Lagrange functions of 
all other members of the hierarchy. Under this assumption, we show that there exists a pluri-Lagrangian 2-form for the hierarchy.

The paper is organized as follows. In Sect. 2 we give a short overview of Lagrangian field theory, recalling some classical notions and definitions. In particular we will provide a formulation of the celebrated Noether theorem, which establishes the relation between conservation laws and variational symmetries. In Sect. 3 we review the notion of continuous 2-dimensional pluri-Lagrangian systems. Section 4 is devoted to new results. It will be proved that from a family of variational symmetries one can construct a pluri-Lagrangian structure. The final Sect. 5 contains three examples which illustrate the theoretical results obtained in Sect. 4.

\section{A short review of Lagrangian field theory}

An exhaustive reference on classical Lagrangian field theory is the book of Olver [20]. The scope of the present section is to recall the main definitions and concepts needed for a self-contained presentation of our results in the next sections.

\subsection{Euler-Lagrange equations}

Since we will work in a multi-time setting we do not restrict our presentation here to fields depending on only two independent variables. Therefore we start by considering a smooth field $u: \mathbb{R}^{N} \rightarrow \mathbb{R}$ depending on $N$ real independent variables $t_{1}, \ldots, t_{N}$.

We will use the multi-index notation for partial derivatives. For any multi-index $I=\left(i_{1}, \ldots, i_{N}\right) \in \mathbb{N}^{N}$ we set

$$
u_{I}=\frac{\partial^{|I|} u}{\left(\partial t_{1}\right)^{i_{1}} \ldots\left(\partial t_{N}\right)^{i_{N}}},
$$

where $|I|=i_{1}+\cdots+i_{N}$ and $u=u\left(t_{1}, \ldots, t_{N}\right)$. The notations $I t_{k}$ and $I t_{k}^{\alpha}$ will represent the multi-indices $\left(i_{1}, \ldots, i_{k}+1, \ldots, i_{N}\right)$ and $\left(i_{1}, \ldots, i_{k}+\alpha, \ldots, i_{N}\right)$ respectively. We will write $k \notin I$ if $i_{k}=0$ and $k \in I$ if $i_{k} \neq 0$.

We will denote by $\mathrm{D}_{i}$ the total derivative with respect to the coordinate direction $t_{i}$,

$$
\mathrm{D}_{i}=\sum_{I \in \mathbb{N}^{N}} u_{I t_{i}} \frac{\partial}{\partial u_{I}}
$$

and by $\mathrm{D}_{I}=\mathrm{D}_{1}^{i_{1}} \ldots \mathrm{D}_{N}^{i_{N}}$ the corresponding higher order derivatives.

The field $u$ can be considered as a section of the trivial bundle $\mathbb{R}^{N} \times \mathbb{R}$. The partial derivatives of $u$ of any order span the infinite jet bundle associated with $\mathbb{R}^{N} \times \mathbb{R}$. We will denote the fiber of the infinite jet bundle by $\mathcal{g}^{\infty}$ and the fiber coordinates by

$$
[u]=\left(u, u_{t_{i}}, u_{t_{i} t_{j}}, \ldots\right)_{i, j, \ldots \in\{1, \ldots, N\}}
$$


A variational problem for a smooth field $u: \mathbb{R}^{N} \rightarrow \mathbb{R}$ is described by a Lagrangian $L: g^{\infty} \rightarrow \mathbb{R}$ and consists in finding the critical points of the action functional

$$
S=\int_{\Gamma} L[u] \mathrm{d} t_{1} \wedge \cdots \wedge \mathrm{d} t_{N}
$$

where $\Gamma \subset \mathbb{R}^{N}$ is some bounded region. In other words, we look for fields $u$ such that for all fields $v$ such that $v$ and its derivatives vanish at the boundary of $\Gamma$, there holds

$$
\left.\frac{\mathrm{d}}{\mathrm{d} \varepsilon}\right|_{\varepsilon=0} \int_{\Gamma} L[u+\varepsilon v] \mathrm{d} t_{1} \wedge \cdots \wedge \mathrm{d} t_{N}=0 .
$$

Concretely, we will be interested in variational problems for fields $u: \mathbb{R}^{2} \rightarrow \mathbb{R}$. Therefore, let us fix $N=2$ and write explicitly the variational equations governing the evolution of $u$. In this case the action functional over some bounded region $\Gamma \subset \mathbb{R}^{2}$ is

$$
S=\int_{\Gamma} L[u] \mathrm{d} t_{1} \wedge \mathrm{d} t_{2}
$$

The field $u$ is a solution to the variational problem, i.e., a critical point for the action $S$, if and only if

$$
\frac{\delta L}{\delta u}=\sum_{\alpha, \beta \geqslant 0}(-1)^{\alpha+\beta} \mathrm{D}_{1}^{\alpha} \mathrm{D}_{2}^{\beta}\left(\frac{\partial L}{\partial u_{t_{1}^{\alpha} t_{2}^{\beta}}}\right)=0
$$

where the left-hand side is called the variational derivative of L. Equation (2) gives rise to a variational PDE, called Euler-Lagrange equation. Note that if the Lagrangian depends on the $n$-th order jet, i.e., on derivatives of $u$ up to order $n$, then the EulerLagrange equation depends on the jet of order $2 n$. If a given 2-dimensional PDE can be written as in equation (2) for some Lagrangian $L$, then we say that this PDE has a variational (or Lagrangian) structure.

Of course, the Euler-Lagrange equation (2) admits a straightforward generalization for the case of a field $u: \mathbb{R}^{N} \rightarrow \mathbb{R}$ for $N>2$.

Example 2.1 The Korteweg-de Vries (KdV) equation

$$
w_{2}=w_{111}+6 w w_{1}
$$

where $w_{i}$ is shorthand notation for the derivative $w_{t_{i}}$, can be put into a variational form by introducing the potential $u=w_{1}$. The corresponding equation is

$$
u_{12}=u_{1111}+6 u_{1} u_{11}
$$

Its variational structure comes from the Lagrangian

$$
L[u]=\frac{1}{2} u_{1} u_{2}-u_{1}^{3}-\frac{1}{2} u_{1} u_{111}
$$


Indeed, critical points of the action (1) are characterized by the Euler-Lagrange equation

$$
\begin{aligned}
0=\frac{\delta L}{\delta u} & =-\mathrm{D}_{1} \frac{\partial L}{\partial u_{1}}-\mathrm{D}_{1}^{3} \frac{\partial L}{\partial u_{111}}-\mathrm{D}_{2} \frac{\partial L}{\partial u_{2}} \\
& =\left(-\frac{1}{2} u_{12}+6 u_{1} u_{11}+\frac{1}{2} u_{1111}\right)+\frac{1}{2} u_{1111}-\frac{1}{2} u_{12} \\
& =-u_{12}+u_{1111}+6 u_{1} u_{11} .
\end{aligned}
$$

\subsection{Variational symmetries and Noether's theorem}

Let $N=2$. A vertical generalized vector field on $\mathbb{R}^{2} \times \mathbb{R}$ is a vector field of the form $Q \partial_{u}$, where $Q: \jmath^{\infty} \rightarrow \mathbb{R}$. It is called vertical because it does not contain any $\partial_{t_{i}}$ and generalized because $Q$ depends on derivatives of $u$, not just on $u$ itself. The prolongation of $Q \partial_{u}$ is a vector field on $g^{\infty}$ defined as

$$
\operatorname{pr}\left(Q \partial_{u}\right)=\sum_{I \in \mathbb{N}^{2}}\left(\mathrm{D}_{I} Q\right) \frac{\partial}{\partial u_{I}}
$$

A vector field $Q \partial_{u}$ is called a variational symmetry of a Lagrangian $L: g^{\infty} \rightarrow \mathbb{R}$ if its prolongation $\operatorname{pr}\left(Q \partial_{u}\right)$ satisfies

$$
\operatorname{pr}\left(Q \partial_{u}\right) L=\mathrm{D}_{1} F_{1}+\mathrm{D}_{2} F_{2}
$$

for some functions $F_{1}, F_{2}: g^{\infty} \rightarrow \mathbb{R}$. The pair $\left(F_{1}, F_{2}\right)$ is called the flux of the variational symmetry.

A conservation law for $L$ is a triple of functions $J_{1}, J_{2}, Q: J^{\infty} \rightarrow \mathbb{R}$ that satisfy

$$
\mathrm{D}_{1} J_{1}+\mathrm{D}_{2} J_{2}=-Q \frac{\delta L}{\delta u}
$$

If equation (4) holds true, the pair $J=\left(J_{1}, J_{2}\right)$ is called the conserved current and $Q$ the characteristic of the conservation law. On solutions of the Euler-Lagrange equations (2) the conserved current $J$ is divergence-free, hence its name.

The famous Noether's theorem [18] establishes a one-to-one correspondence between conservation laws and variational symmetries.

Theorem 2.2 Let $Q \partial_{u}$ be a variational symmetry of $L$. Then

$$
\begin{aligned}
& J_{1}[u]=\sum_{I \not \ngtr t_{2}}\left(\left(\mathrm{D}_{I} Q\right) \frac{\delta L}{\delta u_{I t_{1}}}\right)+\frac{1}{2} \sum_{I} \mathrm{D}_{2}\left(\left(\mathrm{D}_{I} Q\right) \frac{\delta L}{\delta u_{I t_{1} t_{2}}}\right)-F_{1}[u], \\
& J_{2}[u]=\sum_{I \not \ngtr t_{1}}\left(\left(\mathrm{D}_{I} Q\right) \frac{\delta L}{\delta u_{I t_{2}}}\right)+\frac{1}{2} \sum_{I} \mathrm{D}_{1}\left(\left(\mathrm{D}_{I} Q\right) \frac{\delta L}{\delta u_{I t_{1} t_{2}}}\right)-F_{2}[u],
\end{aligned}
$$


define the components of the conserved current of a conservation law, where the pair of functions $\left(F_{1}, F_{2}\right)$ is the flux, as in equation (3).

Conversely, given a conserved current $\left(J_{1}, J_{2}\right)$, equations (5) and (6) define the flux $\left(F_{1}, F_{2}\right)$ of a variational symmetry.

Note that equations (5) and (6) contain variational derivatives with respect to partial derivatives of $u$ :

$$
\frac{\delta L}{\delta u_{I}}=\sum_{\alpha, \beta \geqslant 0}(-1)^{\alpha+\beta} \mathrm{D}_{1}^{\alpha} \mathrm{D}_{2}^{\beta} \frac{\partial L}{\partial u_{I t_{1}^{\alpha} t_{2}^{\beta}}}
$$

We also observe that $J_{1}$ and $J_{2}$ can be alternatively written as

$$
\begin{aligned}
& J_{1}[u]=\sum_{\alpha \geqslant 0}\left(\left(\mathrm{D}_{1}^{\alpha} Q\right) \frac{\delta L}{\delta u_{t_{1}^{\alpha+1}}}\right)+\frac{1}{2} \sum_{\alpha \geqslant 0} \sum_{\beta \geqslant 0} \mathrm{D}_{2}\left(\left(\mathrm{D}_{1}^{\alpha} \mathrm{D}_{2}^{\beta} Q\right) \frac{\delta L}{\delta u_{t_{1}^{\alpha+1} t_{2}^{\beta+1}}}\right)-F_{1}[u], \\
& J_{2}[u]=\sum_{\beta \geqslant 0}\left(\left(\mathrm{D}_{2}^{\beta} Q\right) \frac{\delta L}{\delta u_{t_{2}^{\beta+1}}}\right)+\frac{1}{2} \sum_{\alpha \geqslant 0} \sum_{\beta \geqslant 0} \mathrm{D}_{1}\left(\left(\mathrm{D}_{1}^{\alpha} \mathrm{D}_{2}^{\beta} Q\right) \frac{\delta L}{\delta u_{t_{1}^{\alpha+1} t_{2}^{\beta+1}}}\right)-F_{2}[u] .
\end{aligned}
$$

Proof of Theorem 2.2 The key point of the proof consists in the integration by parts of

$$
\operatorname{pr}\left(Q \partial_{u}\right) L=\sum_{I}\left(\mathrm{D}_{I} Q\right) \frac{\partial L}{\partial u_{I}}
$$

i.e., to write it in the form

$$
\operatorname{pr}\left(Q \partial_{u}\right) L=Q \frac{\delta L}{\delta u}+\mathrm{D}_{1}(\cdots)+\mathrm{D}_{2}(\cdots) .
$$

To perform the full calculation, observe that

$$
\frac{\partial L}{\partial u_{I}}=\frac{\delta L}{\delta u_{I}}+\mathrm{D}_{1} \frac{\delta L}{\delta u_{I t_{1}}}+\mathrm{D}_{2} \frac{\delta L}{\delta u_{I t_{2}}}+\mathrm{D}_{1} \mathrm{D}_{2} \frac{\delta L}{\delta u_{I t_{1} t_{2}}},
$$

hence

$$
\begin{aligned}
\operatorname{pr}\left(Q \partial_{u}\right) L=\sum_{I}\left(\mathrm{D}_{I} Q\right)\left(\frac{\delta L}{\delta u_{I}}\right. & \left.+\mathrm{D}_{1} \frac{\delta L}{\delta u_{I t_{1}}}+\mathrm{D}_{2} \frac{\delta L}{\delta u_{I t_{2}}}+\mathrm{D}_{1} \mathrm{D}_{2} \frac{\delta L}{\delta u_{I t_{1} t_{2}}}\right) \\
=\sum_{I}\left(\left(\mathrm{D}_{I t_{1} t_{2}} Q\right)+\right. & \left.\left(\mathrm{D}_{I t_{2}} Q\right) \mathrm{D}_{1}+\left(\mathrm{D}_{I t_{1}} Q\right) \mathrm{D}_{2}+\left(\mathrm{D}_{I} Q\right) \mathrm{D}_{1} \mathrm{D}_{2}\right) \frac{\delta L}{\delta u_{I t_{1} t_{2}}} \\
& +\sum_{I \not \ngtr t_{2}}\left(\left(\mathrm{D}_{I t_{1}} Q\right)+\left(\mathrm{D}_{I} Q\right) \mathrm{D}_{1}\right) \frac{\delta L}{\delta u_{I t_{1}}} \\
& +\sum_{I \not \nexists t_{1}}\left(\left(\mathrm{D}_{I t_{2}} Q\right)+\left(\mathrm{D}_{I} Q\right) \mathrm{D}_{2}\right) \frac{\delta L}{\delta u_{I t_{2}}}+Q \frac{\delta L}{\delta u},
\end{aligned}
$$


where the last term would be a sum over all $I \not \supset t_{1}, t_{2}$, but only the empty multi-index $I=(0,0)$ satisfies this condition. The above equation can be simplified as

$$
\begin{aligned}
\operatorname{pr}\left(Q \partial_{u}\right) L= & \sum_{I} \mathrm{D}_{1} \mathrm{D}_{2}\left(\left(\mathrm{D}_{I} Q\right) \frac{\delta L}{\delta u_{I t_{1} t_{2}}}\right) \\
& +\sum_{I \not \nexists t_{2}} \mathrm{D}_{1}\left(\left(\mathrm{D}_{I} Q\right) \frac{\delta L}{\delta u_{I t_{1}}}\right)+\sum_{I \not \nexists t_{1}} \mathrm{D}_{2}\left(\left(\mathrm{D}_{I} Q\right) \frac{\delta L}{\delta u_{I t_{2}}}\right)+Q \frac{\delta L}{\delta u} \\
= & \mathrm{D}_{1}\left(J_{1}+F_{1}\right)+\mathrm{D}_{2}\left(J_{2}+F_{2}\right)+Q \frac{\delta L}{\delta u} .
\end{aligned}
$$

It follows that equations (3) and (4) are equivalent. Hence if $Q \partial_{u}$ is a variational symmetry, then equations (5)-(6) define a conserved current.

Example 2.3 Consider again the $\mathrm{KdV}$ equation

$$
u_{12}=u_{1111}+6 u_{1} u_{11}
$$

and its Lagrangian

$$
L[u]=\frac{1}{2} u_{1} u_{2}-u_{1}^{3}-\frac{1}{2} u_{1} u_{111}
$$

As before, indices denote derivatives with respect to the corresponding time variables, e.g. $u_{12}=u_{t_{1} t_{2}}$. We present two variational symmetries of this equation and their associated conservation laws:

(a) The generalized vector field $Q \partial_{u}$ with $Q[u]=u_{1}$ corresponds to a translation in the $t_{1}$-direction. Indeed,

$$
\operatorname{pr}\left(Q \partial_{u}\right) L=u_{1} \frac{\partial L}{\partial u}+u_{11} \frac{\partial L}{\partial u_{1}}+u_{111} \frac{\partial L}{\partial u_{11}}+u_{1111} \frac{\partial L}{\partial u_{111}}+u_{12} \frac{\partial L}{\partial u_{2}}=\mathrm{D}_{1} L,
$$

hence $Q \partial_{u}$ is a variational symmetry with flux

$$
\left(F_{1}[u], F_{2}[u]\right)=(L[u], 0) .
$$

Corresponding to this variational symmetry we find the conservation law

$$
-Q[u] \frac{\delta L}{\delta u}=-u_{1}\left(-u_{12}+6 u_{1} u_{11}+u_{1111}\right)=\mathrm{D}_{1} J_{1}+\mathrm{D}_{2} J_{2},
$$

with

$$
\begin{aligned}
& J_{1}[u]=u_{1} \frac{\delta L}{\delta u_{1}}+u_{11} \frac{\delta L}{\delta u_{11}}+u_{111} \frac{\delta L}{\delta u_{111}}-F_{1}[u]=-2 u_{1}^{3}-u_{1} u_{111}+\frac{1}{2} u_{11}^{2}, \\
& J_{2}[u]=u_{1} \frac{\delta L}{\delta u_{2}}-F_{2}[u]=\frac{1}{2} u_{1}^{2} .
\end{aligned}
$$


This in turn implies the conservation of momentum:

$$
\mathrm{D}_{2} \int \frac{1}{2} u_{1}^{2} \mathrm{~d} t_{1}=0
$$

(b) The generalized vector field $Q \partial_{u}$ with

$$
Q[u]=10 u_{1}^{3}+5 u_{11}^{2}+10 u_{1} u_{111}+u_{11111} .
$$

Indeed,

$$
\begin{aligned}
& \operatorname{pr}\left(Q \partial_{u}\right) L \\
& \quad=Q \frac{\partial L}{\partial u}+\left(\mathrm{D}_{1} Q\right) \frac{\partial L}{\partial u_{1}}+\left(\mathrm{D}_{1}^{2} Q\right) \frac{\partial L}{\partial u_{11}}+\left(\mathrm{D}_{1}^{3} Q\right) \frac{\partial L}{\partial u_{111}}+\left(\mathrm{D}_{2} Q\right) \frac{\partial L}{\partial u_{2}} \\
& \quad=\mathrm{D}_{1} F_{1}+\mathrm{D}_{2} F_{2},
\end{aligned}
$$

with

$$
\begin{aligned}
F_{1}[u]= & -18 u_{1}^{5}-15 u_{1}^{2} u_{11}^{2}-45 u_{1}^{3} u_{111}+5 u_{1}^{3} u_{2}+4 u_{11}^{2} u_{111}-18 u_{1} u_{111}^{2} \\
& -4 u_{1} u_{11} u_{1111}-8 u_{1}^{2} u_{11111}-10 u_{1} u_{11} u_{12}+\frac{5}{2} u_{11}^{2} u_{2}+5 u_{1} u_{111} u_{2} \\
& +\frac{1}{2} u_{1111}^{2}-u_{111} u_{1111}+\frac{1}{2} u_{11} u_{111111}-\frac{1}{2} u_{1} u_{111111}+u_{111} u_{112} \\
& -u_{1111} u_{12}+\frac{1}{2} u_{11111} u_{2}, \\
F_{2}[u]= & \frac{5}{2} u_{1}^{4}+\frac{15}{2} u_{1} u_{11}^{2}+5 u_{1}^{2} u_{111}-\frac{1}{2} u_{111}^{2}+\frac{1}{2} u_{1} u_{11111} .
\end{aligned}
$$

The corresponding conservation law is

$$
-Q[u] \frac{\delta L}{\delta u}=\mathrm{D}_{1} J_{1}+\mathrm{D}_{2} J_{2},
$$

with

$$
\begin{aligned}
J_{1}[u]= & Q \frac{\delta L}{\delta u_{1}}+\left(\mathrm{D}_{1} Q\right) \frac{\delta L}{\delta u_{11}}+\left(\mathrm{D}_{11} Q\right) \frac{\delta L}{\delta u_{111}}-F_{1} \\
= & -12 u_{1}^{5}-15 u_{1}^{2} u_{11}^{2}-10 u_{1}^{3} u_{111}+u_{11}^{2} u_{111}-2 u_{1} u_{111}^{2}-6 u_{1} u_{11} u_{1111} \\
& +10 u_{1} u_{11} u_{12}-\frac{1}{2} u_{1111}^{2}-u_{111} u_{112}+u_{1111} u_{12}
\end{aligned}
$$

and

$$
J_{2}[u]=Q \frac{\delta L}{\delta u_{2}}-F_{2}[u]=\frac{5}{2} u_{1}^{4}-5 u_{1} u_{11}^{2}+\frac{1}{2} u_{111}^{2}
$$




\section{Pluri-Lagrangian field theory}

In this section we briefly review the main concepts of pluri-Lagrangian field theory. For further details see [26-28].

\subsection{Integrable hierarchies of PDEs}

One of the defining features of an integrable PDE is that it possesses an infinite amount of symmetries and, correspondingly, an infinite amount of conservation laws. These symmetries define a family of PDEs that commute with the original one.

Let us illustrate the concept of commuting PDEs on the basis of our leading example.

Example 3.1 In Example 2.3 (b) we proved that the generalized vector field $Q \partial_{u}$, with

$$
Q[u]=10 u_{1}^{3}+5 u_{11}^{2}+10 u_{1} u_{111}+u_{11111},
$$

is a variational symmetry of the $\mathrm{KdV}$ equation

$$
u_{12}=u_{111}+6 u_{1} u_{11}=0 .
$$

If we introduce a third independent variable $t_{3}$, we can define the PDE

$$
u_{3}=10 u_{1}^{3}+5 u_{11}^{2}+10 u_{1} u_{111}+u_{11111},
$$

which commutes with the KdV equation itself. This means that both ways of calculating the mixed derivative $u_{123}$ agree on solutions:

$$
\begin{aligned}
\mathrm{D}_{3} u_{12}= & \mathrm{D}_{3}\left(u_{1111}+6 u_{1} u_{11}\right) \\
= & 540 u_{1}^{2} u_{11}^{2}+180 u_{1}^{3} u_{111}+480 u_{11}^{2} u_{111}+300 u_{1} u_{111}^{2}+480 u_{1} u_{11} u_{1111} \\
& \quad+90 u_{1}^{2} u_{11111}+70 u_{1111}^{2}+110 u_{111} u_{11111}+56 u_{11} u_{111111} \\
& \quad+16 u_{1} u_{1111111}+u_{11111111}
\end{aligned}
$$

Since symmetries lead to commuting equations, a natural perspective on an integrable PDE is to consider it as one equation belonging to an infinite integrable hierarchy, i.e., an infinite set of integrable PDEs such that any two systems in this set are compatible. Such hierarchies are usually generated by recursion operators or master symmetries $[10,17,20]$.

\subsection{Pluri-Lagrangian problems}

Let us focus on $(1+1)$-dimensional PDEs. A finite number of equations from a hierarchy can be embedded in a higher-dimensional multi-time, where they share a common 
space direction, say $t_{1}=x$, but each equation has its own time coordinate, $t_{2}, t_{3}, \ldots$ Formally, we can embed the whole hierarchy into an infinite-dimensional space in the same way.

In the classical variational description of $(1+1)$-dimensional PDEs, we integrate a Lagrange function over (an open subset of) the 2-dimensional space-time. A variational structure of a hierarchy of such PDEs should include the classical variational description of each individual equation, i.e., integration over a 2-dimensional subspace. Therefore, it is natural for the role of a Lagrange function to be played by a differential 2-form.

Let $\mathcal{L} \in \Omega^{2}\left(\mathbb{R}^{N}\right)$ be a 2 -form depending on the infinite jet of a smooth field $u: \mathbb{R}^{N} \rightarrow \mathbb{R}$, i.e.,

$$
\mathcal{L}[u]=\sum_{i<j} L_{i j}[u] \mathrm{d} t_{i} \wedge \mathrm{d} t_{j}
$$

with $L_{i j}: g^{\infty} \rightarrow \mathbb{R}$. We say that $u$ solves the pluri-Lagrangian problem for $\mathcal{L}$ if for any 2-dimensional submanifold $\Gamma \subset \mathbb{R}^{N}$ and for any infinitesimal variation $v\left(t_{1}, \ldots, t_{N}\right) \partial_{u}$ of $u$, where $v: \mathbb{R}^{N} \rightarrow \mathbb{R}$ and all its derivatives vanish at the boundary of $\Gamma$, we have

$$
\left.\frac{\mathrm{d}}{\mathrm{d} \varepsilon}\right|_{\varepsilon=0} \int_{\Gamma} \mathcal{L}[u+\varepsilon v]=0 .
$$

This can also be written as

$$
\int_{\Gamma} \operatorname{pr}\left(v \partial_{u}\right) \mathcal{L}[u]=0
$$

where the vertical vector field $\operatorname{pr}\left(v \partial_{u}\right)=\sum_{I} v_{I} \frac{\partial}{\partial u_{I}}$ acts on the coefficients of $\mathcal{L}[u]$, i.e.,

$$
\operatorname{pr}\left(v \partial_{u}\right) \mathcal{L}[u]=\sum_{i<j} \sum_{I} v_{I} \frac{\partial L_{i j}[u]}{\partial u_{I}} \mathrm{~d} t_{i} \wedge \mathrm{d} t_{j} .
$$

The equations that characterize solutions to the pluri-Lagrangian problem are called multi-time Euler-Lagrange equations. They were derived in [27] and state that, for all $i, j, k \in\{1, \ldots, N\}$, there holds:

$$
\begin{aligned}
\text { for all } I \not \nexists t_{i}, t_{j}: & \frac{\delta_{i j} L_{i j}}{\delta u_{I}}=0, \\
\text { for all } I \not \nexists t_{i}: & \frac{\delta_{i j} L_{i j}}{\delta u_{I t_{j}}}=\frac{\delta_{i k} L_{i k}}{\delta u_{I t_{k}}}, \\
\text { for all } I: & \frac{\delta_{i j} L_{i j}}{\delta u_{I t_{i} t_{j}}}+\frac{\delta_{j k} L_{j k}}{\delta u_{I t_{j} t_{k}}}+\frac{\delta_{k i} L_{k i}}{\delta u_{I t_{k} t_{i}}}=0,
\end{aligned}
$$


where

$$
\frac{\delta_{i j} L_{i j}}{\delta u_{I}}=\sum_{\alpha, \beta \geqslant 0}(-1)^{\alpha+\beta} \mathrm{D}_{i}^{\alpha} \mathrm{D}_{j}^{\beta}\left(\frac{\partial L_{i j}}{\partial u_{I i^{\alpha} j}}\right)
$$

is the variational derivative in the $\left(t_{i}, t_{j}\right)$-plane. Note that the multi-time EulerLagrange equations contain the classical Euler-Lagrange equations in each $\left(t_{i}, t_{j}\right)$ plane (8), where derivatives with respect to other times are considered as additional components of the field, plus additional equations (9)-(10) coming from choices of $\Gamma$ that are not coordinate planes.

In the present work, we will use a different property to recognize solutions to the pluri-Lagrangian problem. There is a remarkable relation between the pluriLagrangian problem and the property that the 2 -form $\mathcal{L}$ is closed on solutions $u$ to the hierarchy. In fact, this closedness property is often considered to be the fundamental property of the Lagrangian theory of integrable hierarchies [2,13-16,32,33]. When this point of view is taken, the term "Lagrangian multiform" is more commonly used than "pluri-Lagrangian".

Here, we show that a slightly weaker property of the 2-form is a sufficient condition for a solution to the pluri-Lagrangian problem.

Theorem 3.2 Consider a 2-form $\mathcal{L}$ and a hierarchy of commuting PDEs

$$
u_{i}=Q_{i}[u], \quad i=2, \ldots, N
$$

with $Q_{i}: \mathcal{J}^{\infty} \rightarrow \mathbb{R}$. If the exterior derivative of $\mathcal{L}$ is constant up to a term that attains a double zero on solutions of (12), i.e., if

$$
\mathrm{d} \mathcal{L}=\gamma+\sum_{I, J} \sum_{i, j} \omega_{i, j}^{I, J} \mathrm{D}_{I}\left(u_{i}-Q_{i}\right) \mathrm{D}_{J}\left(u_{j}-Q_{j}\right)
$$

for some $\mathrm{g}^{\infty}$-dependent 3-forms $\omega_{i, j}^{I, J}$ and a 3-form $\gamma$ that does not depend on $u$ or its derivatives, then all solutions $u: \mathbb{R}^{N} \rightarrow \mathbb{R}$ to the hierarchy (12) also solve the pluri-Lagrangian problem for $\mathcal{L}$.

Strictly speaking, the assumption that the PDEs (12) commute can be dropped from this theorem. If they do not commute then there will usually be no non-trivial solutions $u: \mathbb{R}^{N} \rightarrow \mathbb{R}$ to all PDEs simultaneously, so in this case the theorem would be of very limited relevance.

Proof of Theorem 3.2 Let $u$ be a solution to the hierarchy and $\Gamma=\partial B$ a surface defined as the boundary of a 3-manifold $B$. It is sufficient to show that the pluri-Lagrangian property holds on such surfaces. Indeed, without loss of generality we can require variations to be supported on small open subsets and for any sufficiently small open subset $\Gamma^{\prime}$ of a given surface, one can find a 3-manifold such that $\Gamma^{\prime}$ is contained in its boundary. 
As a consequence of the assumption on $\mathcal{L}$ there holds for any variation $v: \mathbb{R}^{N} \rightarrow \mathbb{R}$ that

$$
\operatorname{pr}\left(v \partial_{u}\right) \mathrm{d} \mathcal{L}[u]=\left.\frac{\mathrm{d}}{\mathrm{d} \varepsilon}\right|_{\varepsilon=0} \mathrm{~d} \mathcal{L}[u+\varepsilon v]=0 .
$$

Therefore

$$
\left.\frac{\mathrm{d}}{\mathrm{d} \varepsilon}\right|_{\varepsilon=0} \int_{\Gamma} \mathcal{L}[u+\varepsilon v]=\left.\frac{\mathrm{d}}{\mathrm{d} \varepsilon}\right|_{\varepsilon=0} \int_{B} \mathrm{~d} \mathcal{L}[u+\varepsilon v]=0,
$$

hence the action integral over any surface $\Gamma$ is critical with respect to variations of $u$.

There are strong indications that the existence of a pluri-Lagrangian structure is deeply connected to integrability. One such indication comes from within the theory: the multi-time Euler-Lagrange equations are highly overdetermined. Hence if nontrivial solutions exist, then we are dealing with a system with remarkable properties. Other indications are connections to different notions of integrability, including Hamiltonian formulations [26,28] and Lax pairs [24], even though these connections have not yet been studied in full detail.

Despite some recent discoveries, relatively few examples of pluri-Lagrangian hierarchies of PDEs are known. To our knowledge, the list is limited to the potential $\mathrm{KdV}$ equation [27] and several related hierarchies obtained as continuum limits from lattice equations [29,30], as well as (a matrix-valued generalization of) the AKNS system [24]. The goal of this paper is to establish a construction of a pluri-Lagrangian 2-form for a given hierarchy of $(1+1)$-dimensional PDEs, assuming we know classical Lagrange functions for the individual equations. Furthermore, we will assume that the vector field associated to each of the PDEs is a variational symmetry for the Lagrangians of the rest of the hierarchy. This assumption can be thought of as the Lagrangian analogue to commuting Hamiltonian flows.

\section{From variational symmetries to a pluri-Lagrangian 2-form}

We will take $t_{1}=x$ to be the space coordinate. Then we can take the coefficients $L_{1 j}$ of the pluri-Lagrangian 2-form (7) to be classical Lagrangians for the individual equations of the hierarchy. However, the coefficients $L_{i j}$ with $i, j>1$ do not have an interpretation in a classical variational principle. It is not obvious under which conditions suitable $L_{i j}$ exist, such that the given hierarchy solves the pluri-Lagrangian problem for the 2-form. Below we will give an answer to this question for a large class of Lagrangians.

For a hierarchy of evolutionary equations,

$$
u_{i}=Q_{i}\left(u_{1}, u_{11}, \ldots\right), \quad i=2, \ldots, N
$$


it is a reasonable assumption that the corresponding Lagrangians do not contain second or higher derivatives with respect to the time variable. Similarly, we will assume that the Lagrangian does not contain products of time-derivatives. Suppose we have a family of Lagrangians $L_{1 i}$ for $i=2, \ldots, N$ satisfying these assumptions:

$$
L_{1 i}[u]=p\left(u, u_{1}, u_{11}, \ldots\right) u_{i}-h_{i}\left(u, u_{1}, u_{11}, \ldots\right) .
$$

Here $p$ and $h$ are two arbitrary functions of their arguments. In particular the term $p\left(u, u_{1}, u_{11}, \ldots\right) u_{i}$ plays the role of a kinetic energy. Note that we are not including mixed derivatives, $u_{1 i}, u_{11 i}, \ldots$ This does not restrict generality, because if a Lagrangian depends linearly on such derivatives, then we can integrate by parts to get an equivalent Lagrangian of the form (14). Furthermore, note that the factor $p\left(u, u_{1}, u_{11}, \ldots\right)$ in the kinetic term of $L_{1 i}[u]$ is the same for all $i$. This is a direct consequence of the multi-time Euler-Lagrange equations of type (9).

The Euler-Lagrange equations (2) of the Lagrangians (14) will not be evolutionary. Instead we assume that the Euler-Lagrange equations are differential consequences of the hierarchy (13), i.e., equations of the form

$$
\mathcal{E}_{p}\left(u_{i}-Q_{i}\left(u_{1}, u_{11}, \ldots\right)\right)=0
$$

where $\varepsilon_{p}$ is some differential operator, depending on the kinetic term of the Lagrangians. In the case of the KdV hierarchy we have $\varepsilon_{p}=\mathrm{D}_{1}$, see Example 2.1.

Assume that the prolonged vector fields $\mathfrak{D}_{i}=\operatorname{pr}\left(Q_{i} \partial_{u}\right)$, corresponding to the equations of the hierarchy, commute pairwise and are variational symmetries of the $L_{1 j}$ :

$$
\mathfrak{D}_{i} L_{1 j}=\mathrm{D}_{1} A_{i j}+\mathrm{D}_{j} B_{i j}
$$

for some functions $A_{i j}, B_{i j}: \mathcal{J}^{\infty} \rightarrow \mathbb{R}$. If we consider only those terms that contain a $t_{j}$-derivative, what remains of equation (15) is of the form

$$
\mathfrak{D}_{i}\left(p u_{j}\right)=\mathrm{D}_{1} \bar{A}_{i j}\left(u, u_{1}, u_{j}, \ldots\right)+\mathrm{D}_{j} B_{i j}\left(u, u_{1}, u_{11}, \ldots\right)
$$

for some function $\bar{A}_{i j}: \jmath^{\infty} \rightarrow \mathbb{R}$. This is an algebraic identity (as opposed to an equality on solutions), hence we can replace $t_{j}$-derivatives by new dependent variables, e.g. $u_{j}$ by a field denoted by $u_{t}$. We find

$$
\mathfrak{D}_{i}\left(p u_{t}\right)=\mathrm{D}_{1} \bar{A}_{i j}\left(u, u_{1}, u_{t}, \ldots\right)+\mathrm{D}_{k} B_{i j}\left(u, u_{1}, u_{11}, \ldots\right) .
$$

Since the left-hand side of this equation is independent of $j$, we can choose $\bar{A}_{i j}$ and $B_{i j}$ independent of $j$ as well. In particular, we can write $B_{i j}=B_{i}$ and get

$$
\mathfrak{D}_{i} L_{1 j}=\mathrm{D}_{1} A_{i j}+\mathrm{D}_{j} B_{i} .
$$

Note that $A_{i j}, B_{i}: \jmath^{\infty} \rightarrow \mathbb{R}$ are only defined up to a constant, hence we can choose them to be zero on the zero field: $A_{i j}[0]=B_{i}[0]=0$. 
Lemma 4.1 For Lagrangians of the form (14) with commuting variational symmetries (15), there exist functions $F_{i j}: g^{\infty} \rightarrow \mathbb{R}:[u] \mapsto F_{i j}\left(u, u_{1}, u_{11}, \ldots\right)$, that do not depend on any time-derivatives, such that

$$
\mathrm{D}_{1} F_{i j}=\mathrm{D}_{i} L_{1 j}-\mathrm{D}_{j} L_{1 i}
$$

on solutions of the hierarchy (13).

Proof Since the variational symmetries $\mathfrak{D}_{i}=\operatorname{pr}\left(Q_{i} \partial_{u}\right)$ commute, we have for any $k \neq i, j$,

$$
\begin{aligned}
0 & =\left[\mathfrak{D}_{i}, \mathfrak{D}_{j}\right] L_{1 k} \\
& =\mathrm{D}_{1}\left(\mathfrak{D}_{i} A_{j k}-\mathfrak{D}_{j} A_{i k}\right)+\mathrm{D}_{k}\left(\mathfrak{D}_{i} B_{j}-\mathfrak{D}_{j} B_{i}\right) .
\end{aligned}
$$

Now let $u$ be an arbitrary compactly supported smooth field. Then

$$
\begin{aligned}
0 & =\int_{-\infty}^{\infty} \mathrm{D}_{1}\left(\mathfrak{D}_{i} A_{j k}-\mathfrak{D}_{j} A_{i k}\right)+\mathrm{D}_{k}\left(\mathfrak{D}_{i} B_{j}-\mathfrak{D}_{j} B_{i}\right) \mathrm{d} t_{1} \\
& =\int_{-\infty}^{\infty} \mathrm{D}_{k}\left(\mathfrak{D}_{i} B_{j}-\mathfrak{D}_{j} B_{i}\right) \mathrm{d} t_{1} \\
& =\mathrm{D}_{k} \int_{-\infty}^{\infty}\left(\mathfrak{D}_{i} B_{j}-\mathfrak{D}_{j} B_{i}\right) \mathrm{d} t_{1}
\end{aligned}
$$

Since $u$ and in particular its $t_{k}$-derivatives are arbitrary, it follows that $\mathfrak{D}_{i} B_{j}-\mathfrak{D}_{j} B_{i}$ is a null Lagrangian. This implies (see e.g. [20, Theorem 4.7]) that there exists a function $G_{i j}: \mathcal{J}^{\infty} \rightarrow \mathbb{R}$ such that

$$
\mathfrak{D}_{i} B_{j}-\mathfrak{D}_{j} B_{i}=\mathrm{D}_{1}\left(G_{i j}\right) \text {. }
$$

Hence with $F_{i j}=G_{i j}+A_{i j}-A_{j i}$ we find that, on solutions of the hierarchy (13),

$$
\begin{aligned}
\mathfrak{D}_{i} L_{1 j}-\mathfrak{D}_{j} L_{1 i} & =\mathrm{D}_{1} A_{i j}+\mathrm{D}_{j} B_{i}-\mathrm{D}_{1} A_{j i}-\mathrm{D}_{i} B_{j} \\
& =\mathrm{D}_{1} A_{i j}+\mathfrak{D}_{j} B_{i}-\mathrm{D}_{1} A_{j i}-\mathfrak{D}_{i} B_{j} \\
& =\mathrm{D}_{1} F_{i j} .
\end{aligned}
$$

Since we are working on solutions of the equations the hierarchy, we can use those equations to eliminate time-derivatives from $F_{i j}$, hence we can assume it depends on the jet as $F_{i j}\left(u, u_{1}, u_{11}, \ldots\right)$.

We now present our main result, which is the analogue in 2-dimensional field theory of [22, Theorem 10]. 
Theorem 4.2 Assume we have Lagrangians of the form (14) with commuting variational symmetries (15). Let

$$
\begin{aligned}
& L_{i j}[u]=\sum_{\alpha \geqslant 0} \frac{\delta_{1 j} L_{1 j}}{\delta u_{t_{1}^{\alpha+1}}} \mathrm{D}_{1}^{\alpha}\left(u_{i}-Q_{i}\right)-\sum_{\alpha \geqslant 0} \frac{\delta_{1 i} L_{1 i}}{\delta u_{t_{1}^{\alpha+1}}} \mathrm{D}_{1}^{\alpha}\left(u_{j}-Q_{j}\right) \\
& +F_{i j}\left(u, u_{1}, u_{11}, \ldots\right) \text {, }
\end{aligned}
$$

where $F_{i j}: g^{\infty} \rightarrow \mathbb{R}$ is as in Lemma 4.1 and the operator $\frac{\delta_{i j}}{\delta}$ is the variational derivative from equation (11). Then every solution of the hierarchy (13) is a critical point of

$$
\mathcal{L}[u]=\sum_{i<j} L_{i j}[u] \mathrm{d} t_{i} \wedge \mathrm{d} t_{j}
$$

in the pluri-Lagrangian sense.

Proof We show that $\mathcal{L}$ is almost-closed in the sense of Theorem 3.2. We start by calculating $\mathrm{D}_{1} L_{i j}$. We have

$$
\begin{aligned}
& \mathrm{D}_{1}\left(\sum_{\alpha \geqslant 0} \frac{\delta_{1 j} L_{1 j}}{\delta u_{t_{1}^{\alpha+1}}} \mathrm{D}_{1}^{\alpha}\left(u_{i}-Q_{i}\right)\right) \\
& =\sum_{\alpha \geqslant 0} \mathrm{D}_{1} \frac{\delta_{1 j} L_{1 j}}{\delta u_{t_{1}^{\alpha+1}}} \mathrm{D}_{1}^{\alpha}\left(u_{i}-Q_{i}\right)+\sum_{\alpha \geqslant 0} \frac{\delta_{1 j} L_{1 j}}{\delta u_{t_{1}^{\alpha+1}}} \mathrm{D}_{1}^{\alpha+1}\left(u_{i}-Q_{i}\right) \\
& =\sum_{\alpha \geqslant 0}\left(\mathrm{D}_{1} \frac{\delta_{1 j} L_{1 j}}{\delta u_{t_{1}^{\alpha+1}}}+\frac{\delta_{1 j} L_{1 j}}{\delta u_{t_{1}^{\alpha}}}\right) \mathrm{D}_{1}^{\alpha}\left(u_{i}-Q_{i}\right)-\frac{\delta_{1 j} L_{1 j}}{\delta u} \mathrm{D}_{1}\left(u_{i}-Q_{i}\right) \\
& =\sum_{\alpha \geqslant 0}\left(\frac{\partial L_{1 j}}{\partial u_{t_{1}^{\alpha}}}-\mathrm{D}_{j} \frac{\delta L_{1 j} L_{1 j}}{\delta u_{t_{1}^{\alpha} t_{j}}}-\mathrm{D}_{1} \mathrm{D}_{j} \frac{\delta_{1 j} L_{1 j}}{\delta u_{t_{1}^{\alpha+1} t_{j}}}\right) \mathrm{D}_{1}^{\alpha}\left(u_{i}-Q_{i}\right) \\
& -\frac{\delta_{1 j} L_{1 j}}{\delta u} \mathrm{D}_{1}\left(u_{i}-Q_{i}\right) .
\end{aligned}
$$

Since $L_{1 j}$ does not depend on any mixed derivatives $u_{t_{1}^{\alpha+1} t_{j}}$, this simplifies to

$$
\begin{aligned}
& \mathrm{D}_{1}\left(\sum_{\alpha \geqslant 0} \frac{\delta_{1 j} L_{1 j}}{\delta u_{t_{1}^{\alpha+1}}} \mathrm{D}_{1}^{\alpha}\left(u_{i}-Q_{i}\right)\right) \\
& =\sum_{\alpha \geqslant 0} \frac{\partial L_{1 j}}{\partial u_{t_{1}^{\alpha}}} \mathrm{D}_{1}^{\alpha}\left(u_{i}-Q_{i}\right)-\mathrm{D}_{j} \frac{\delta_{1 j} L_{1 j}}{\delta u_{t_{j}}}\left(u_{i}-Q_{i}\right)-\frac{\delta_{1 j} L_{1 j}}{\delta u} \mathrm{D}_{1}\left(u_{i}-Q_{i}\right) \\
& \equiv \sum_{\alpha \geqslant 0} \frac{\partial L_{1 j}}{\partial u_{t_{1}^{\alpha}}} \mathrm{D}_{1}^{\alpha}\left(u_{i}-Q_{i}\right)-\left(\mathrm{D}_{j} p\right)\left(u_{i}-Q_{i}\right),
\end{aligned}
$$


where $\equiv$ denotes equality modulo double zeros. Similarly, there holds

$$
\mathrm{D}_{1}\left(\sum_{\alpha \geqslant 0} \frac{\delta_{1 i} L_{1 i}}{\delta u_{t_{1}^{\alpha+1}}} \mathrm{D}_{1}^{\alpha}\left(u_{j}-Q_{j}\right)\right) \equiv \sum_{\alpha \geqslant 0} \frac{\partial L_{1 i}}{\partial u_{t_{1}^{\alpha}}} \mathrm{D}_{1}^{\alpha}\left(u_{j}-Q_{j}\right)-\left(\mathrm{D}_{i} p\right)\left(u_{j}-Q_{j}\right)
$$

Hence

$$
\begin{aligned}
\mathrm{D}_{1} L_{i j} \equiv \sum_{\alpha \geqslant 0} \frac{\partial L_{1 j}}{\partial u_{t_{1}^{\alpha}}} \mathrm{D}_{1}^{\alpha}\left(u_{i}-Q_{i}\right) & -\sum_{\alpha \geqslant 0} \frac{\partial L_{1 i}}{\partial u_{t_{1}^{\alpha}}} \mathrm{D}_{1}^{\alpha}\left(u_{j}-Q_{j}\right) \\
& -\left(\mathrm{D}_{j} p\right)\left(u_{i}-Q_{i}\right)+\left(\mathrm{D}_{i} p\right)\left(u_{j}-Q_{j}\right)+\mathrm{D}_{1} F_{i j} .
\end{aligned}
$$

Using the assumption that the Lagrangians $L_{1 i}$ and $L_{1 j}$ are of the form (14), we can write

$$
\begin{aligned}
& \mathrm{D}_{i} L_{1 j}-\mathfrak{D}_{i} L_{1 j}=p \mathrm{D}_{j}\left(u_{i}-Q_{i}\right)+\sum_{\alpha \geqslant 0} \frac{\partial L_{1 j}}{\partial u_{t_{1}^{\alpha}}} \mathrm{D}_{1}^{\alpha}\left(u_{i}-Q_{i}\right), \\
& \mathrm{D}_{j} L_{1 i}-\mathfrak{D}_{j} L_{1 i}=p \mathrm{D}_{i}\left(u_{j}-Q_{j}\right)+\sum_{\alpha \geqslant 0} \frac{\partial L_{1 i}}{\partial u_{t_{1}^{\alpha}}} \mathrm{D}_{1}^{\alpha}\left(u_{j}-Q_{j}\right),
\end{aligned}
$$

where $\mathfrak{D}_{i}=\operatorname{pr}\left(Q_{i} \partial_{u}\right)$ and $\mathfrak{D}_{j}=\operatorname{pr}\left(Q_{j} \partial_{u}\right)$. Hence

$$
\begin{aligned}
\mathrm{D}_{1} L_{i j}-\mathrm{D}_{i} L_{1 j}+\mathrm{D}_{j} L_{1 i} \equiv-\mathfrak{D}_{i} L_{1 j} & +\mathfrak{D}_{j} L_{1 i}-\left(\mathrm{D}_{j} p\right)\left(u_{i}-Q_{i}\right) \\
& -p \mathrm{D}_{j}\left(u_{i}-Q_{i}\right)+\left(\mathrm{D}_{i} p\right)\left(u_{j}-Q_{j}\right) \\
& +p \mathrm{D}_{i}\left(u_{j}-Q_{j}\right)+\mathrm{D}_{1} F_{i j} .
\end{aligned}
$$

By definition of $F_{i j}$ we have that $\mathrm{D}_{1} F_{i j}-\mathfrak{D}_{i} L_{1 j}+\mathfrak{D}_{j} L_{1 i}=0$ on solutions of (13). Furthermore, the only time derivatives in this expression come from the kinetic parts $p u_{i}$ and $p u_{j}$ of the Lagrangians. Therefore,

$$
\begin{aligned}
& \mathrm{D}_{1} F_{i j}- \mathfrak{D}_{i} L_{1 j}+\mathfrak{D}_{j} L_{1 i} \\
&=-\mathfrak{D}_{i}\left(p u_{j}-p Q_{j}\right)+\mathfrak{D}_{j}\left(p u_{i}-p Q_{i}\right) \\
&=-p \mathfrak{D}_{i}\left(u_{j}-Q_{j}\right)-\left(\mathfrak{D}_{i} p\right)\left(u_{j}-Q_{j}\right) \quad+p \mathfrak{D}_{j}\left(u_{i}-Q_{i}\right)+\left(\mathfrak{D}_{j} p\right)\left(u_{i}-Q_{i}\right) \\
& \equiv-p \mathrm{D}_{i}\left(u_{j}-Q_{j}\right)-\left(\mathrm{D}_{i} p\right)\left(u_{j}-Q_{j}\right) \\
& \quad+p \mathrm{D}_{j}\left(u_{i}-Q_{i}\right)+\left(\mathrm{D}_{j} p\right)\left(u_{i}-Q_{i}\right) .
\end{aligned}
$$

Combining equations (17) and (18) gives

$$
\mathrm{D}_{1} L_{i j}-\mathrm{D}_{i} L_{1 j}+\mathrm{D}_{j} L_{1 i} \equiv 0 \text {. }
$$


Consider three copies of equation (19), each with an additional differentiation:

$$
\begin{aligned}
& \mathrm{D}_{k}\left(\mathrm{D}_{1} L_{i j}-\mathrm{D}_{i} L_{1 j}+\mathrm{D}_{j} L_{1 i}\right) \equiv 0 \\
& \mathrm{D}_{j}\left(\mathrm{D}_{1} L_{i k}-\mathrm{D}_{i} L_{1 k}+\mathrm{D}_{k} L_{1 i}\right) \equiv 0 \\
& \mathrm{D}_{i}\left(\mathrm{D}_{1} L_{j k}-\mathrm{D}_{j} L_{1 k}+\mathrm{D}_{k} L_{1 j}\right) \equiv 0 .
\end{aligned}
$$

A linear combination of these three equations gives us

$$
\mathrm{D}_{1}\left(\mathrm{D}_{k} L_{i j}-\mathrm{D}_{j} L_{i j}+\mathrm{D}_{i} L_{j k}\right) \equiv 0 \text {. }
$$

Since all coefficients are autonomous, this implies that

$$
\mathrm{D}_{k} L_{i j}-\mathrm{D}_{j} L_{i j}+\mathrm{D}_{i} L_{j k} \equiv \text { const. }
$$

Equations (19) and (20) together imply that $\mathcal{L}$ fulfills the conditions of Theorem 3.2, hence every solution of the hierarchy (13) is a critical point of the pluri-Lagrangian problem for $\mathcal{L}$.

Theorem 4.2 and its proof are formulated for scalar systems, but they can be extended to the case of multi-component systems. If $u=\left(u^{1}, \ldots, u^{\ell}\right)$ satisfies the equations $u_{i}^{k}=Q_{i}^{k}$, we construct the Lagrangian coefficients by

$$
\begin{aligned}
& L_{i j}[u]=\sum_{k=1}^{\ell} \sum_{\alpha \geqslant 0} \frac{\delta_{1 j} L_{1 j}}{\delta u_{t_{1}^{\alpha+1}}^{k}} \mathrm{D}_{1}^{\alpha}\left(u_{i}^{k}-Q_{i}^{k}\right)-\sum_{k=1}^{\ell} \sum_{\alpha \geqslant 0} \frac{\delta_{1 i} L_{1 i}}{\delta u_{t_{1}^{\alpha+1}}^{k}} \mathrm{D}_{1}^{\alpha}\left(u_{j}^{k}-Q_{j}^{k}\right) \\
& +F_{i j}\left(u, u_{1}, u_{11}, \ldots\right) \text {. }
\end{aligned}
$$

\section{Examples}

In this last section we discuss three examples. For the first one, the potential Kortewegde Vries hierarchy, a pluri-Lagrangian structure is known in the literature [28]. Our discussion illustrates that this structure can be obtained using Theorem 4.2. The second example is the Nonlinear Schrödinger (NLS) hierarchy. Its pluri-Lagrangian structure can be considered as a special case of the one for the AKNS hierarchy obtained in [24]. The final example is the system consisting of the sine-Gordon and modified $\mathrm{KdV}$ equations, which indicates that the construction of Theorem 4.2 can be adapted to non-evolutionary equations.

The calculations in this section were performed in the SageMath software system [23]. The code is available at [31].

\subsection{Potential KdV hierarchy}

We start with our running example of the Korteweg-de Vries equation. The potential $\mathrm{KdV}$ hierarchy was the first complete hierarchy of PDEs for which a pluri-Lagrangian 
structure was found [27]. Here we show that this structure can also be derived using Theorem 4.2. We present only a minimal example consisting of just the first two equations in the hierarchy,

$$
\begin{aligned}
& u_{2}=3 u_{1}^{2}+u_{111} \\
& u_{3}=10 u_{1}^{3}+5 u_{11}^{2}+10 u_{1} u_{111}+u_{11111}
\end{aligned}
$$

The corresponding Lagrangians are

$$
\begin{aligned}
& L_{12}[u]=\frac{1}{2} u_{1} u_{2}-u_{1}^{3}-\frac{1}{2} u_{1} u_{111}, \\
& L_{13}[u]=\frac{1}{2} u_{1} u_{3}-\frac{5}{2} u_{1}^{4}+5 u_{1} u_{11}^{2}-\frac{1}{2} u_{111}^{2},
\end{aligned}
$$

and have as their Euler-Lagrange equations

$$
\begin{aligned}
& \mathrm{D}_{1}\left(u_{2}-\left(3 u_{1}^{2}+u_{111}\right)\right)=0 \\
& \mathrm{D}_{1}\left(u_{3}-\left(10 u_{1}^{3}+5 u_{11}^{2}+10 u_{1} u_{111}+u_{11111}\right)\right)=0
\end{aligned}
$$

On solutions of the evolutionary equations, there holds

$$
\begin{aligned}
\mathrm{D}_{2} L_{13}-\mathrm{D}_{3} L_{12}=-10 u_{1}^{3} u_{12} & +10 u_{1} u_{11} u_{112}+5 u_{11}^{2} u_{12}+3 u_{1}^{2} u_{13}-u u_{111} u_{1112} \\
& +\frac{1}{2} u_{1} u_{1113}+\frac{1}{2} u_{111} u_{13}-\frac{1}{2} u_{13} u_{2}+\frac{1}{2} u_{12} u_{3} \\
=15 u_{1}^{4} u_{11} & +135 u_{1} u_{11}^{3}+210 u_{1}^{2} u_{11} u_{111}+25 u_{1}^{3} u_{1111} \\
& -18 u_{11} u_{111}^{2}+\frac{15}{2} u_{11}^{2} u_{1111}+34 u_{1} u_{111} u_{1111} \\
& +33 u_{1} u_{11} u_{11111}+\frac{13}{2} u_{1}^{2} u_{11111}+\frac{1}{2} u_{1111} u_{11111} \\
& -u_{111} u_{111111}+\frac{1}{2} u_{1} u_{11111111} .
\end{aligned}
$$

Integrating this gives us

$$
\begin{aligned}
F_{23}\left(u, u_{1}, u_{11}, \ldots\right)= & 3 u_{1}^{5}+\frac{135}{2} u_{1}^{2} u_{11}^{2}+25 u_{1}^{3} u_{111}-\frac{25}{2} u_{11}^{2} u_{111}+7 u_{1} u_{111}^{2} \\
& +20 u_{1} u_{11} u_{1111}+\frac{13}{2} u_{1}^{2} u_{11111}+\frac{1}{2} u_{1111}^{2}-\frac{1}{2} u_{111} u_{11111} \\
& -\frac{1}{2} u_{11} u_{11111}+\frac{1}{2} u_{1} u_{1111111} .
\end{aligned}
$$


Let $Q_{2}$ and $Q_{3}$ be the right-hand sides of equations (21) and (22). Then the remaining terms in equation (16) are

$$
\begin{aligned}
& \frac{\delta_{13} L_{13}}{\delta u_{1}}\left(u_{2}-Q_{2}\right)=\left(\frac{1}{2} u_{3}-10 u_{1}^{3}-5 u_{11}^{2}-10 u_{1} u_{111}-u_{11111}\right) \\
& \cdot\left(u_{2}-3 u_{1}^{2}-u_{111}\right), \\
& \frac{\delta_{13} L_{13}}{\delta u_{11}} \mathrm{D}_{1}\left(u_{2}-Q_{2}\right)=\left(10 u_{1} u_{11}-u_{1111}\right)\left(u_{12}-6 u_{1} u_{11}-u_{1111}\right), \\
& \frac{\delta_{13} L_{13}}{\delta u_{111}} \mathrm{D}_{11}\left(u_{2}-Q_{2}\right)=u_{111}\left(u_{112}-6 u_{1} u_{111}-6 u_{11}^{2}-u_{11111}\right),
\end{aligned}
$$

and

$$
\begin{aligned}
&-\frac{\delta_{12} L_{12}}{\delta u_{1}}\left(u_{3}-Q_{3}\right)=-\left(\frac{1}{2} u_{2}-3 u_{1}^{2}-u_{111}\right) \\
& \cdot\left(u_{3}-10 u_{1}^{3}-5 u_{11}^{2}-10 u_{1} u_{111}-u_{11111}\right)\left.-10 u_{1} u_{1111}-u_{111111}\right) \\
&-\frac{\delta_{12} L_{12}}{\delta u_{11}} \mathrm{D}_{1}\left(u_{3}-Q_{3}\right)=-\frac{1}{2} u_{11}\left(u_{13}-30 u_{1}^{2} u_{11}-20 u_{11} u_{111}\right. \\
&-\frac{\delta_{12} L_{12}}{\delta u_{111}} \mathrm{D}_{11}\left(u_{3}-Q_{3}\right)=\frac{1}{2} u_{1}\left(u_{113}-60 u_{1} u_{11}^{2}-30 u_{1}^{2} u_{111}-20 u_{111}^{2}\right. \\
&\left.-30 u_{11} u_{1111}-10 u_{1} u_{11111}-u_{1111111}\right)
\end{aligned}
$$

Adding everything together, as in equation (16) of Theorem 4.2, we find

$$
\begin{aligned}
L_{23}[u]= & 3 u_{1}^{5}-\frac{15}{2} u_{1}^{2} u_{11}^{2}+10 u_{1}^{3} u_{111}-5 u_{1}^{3} u_{2}+\frac{7}{2} u_{11}^{2} u_{111}+3 u_{1} u_{111}^{2} \\
& -6 u_{1} u_{11} u_{1111}+\frac{3}{2} u_{1}^{2} u_{11111}+10 u_{1} u_{11} u_{12}-\frac{5}{2} u_{11}^{2} u_{2}-5 u_{1} u_{111} u_{2} \\
& +\frac{3}{2} u_{1}^{2} u_{3}-\frac{1}{2} u_{1111}^{2}+\frac{1}{2} u_{111} u_{11111}-u_{111} u_{112}+\frac{1}{2} u_{1} u_{113} \\
& +u_{1111} u_{12}-\frac{1}{2} u_{11} u_{13}-\frac{1}{2} u_{11111} u_{2}+\frac{1}{2} u_{111} u_{3} .
\end{aligned}
$$

Note that the classical Euler-Lagrange equations

$$
\frac{\delta_{12} L_{12}}{\delta u}=0 \text { and } \frac{\delta_{13} L_{13}}{\delta u}=0
$$

yield equations (23)-(24), which are the $t_{1}$-derivatives of the potential $\mathrm{KdV}$ equations (21)-(22). However, the multi-time Euler-Lagrange equations also contain the potential $\mathrm{KdV}$ equations themselves: 


$$
\begin{aligned}
& \frac{\delta_{12} L_{12}}{\delta u_{1}}=-\frac{\delta_{23} L_{23}}{\delta u_{3}} \Longrightarrow \frac{1}{2} u_{2}-3 u_{1}^{2}-u_{111}=-\frac{3}{2} u_{1}^{2}-\frac{1}{2} u_{111} \text {, } \\
& \frac{\delta_{13} L_{13}}{\delta u_{1}}=\frac{\delta_{23} L_{23}}{\delta u_{2}} \Longrightarrow \frac{1}{2} u_{3}-10 u_{1}^{3}-5 u_{11}^{2}-10 u_{1} u_{111}-u_{11111} \\
& =-5 u_{1}^{3}-\frac{5}{2} u_{11}^{2}-5 u_{1} u_{111}-\frac{1}{2} u_{11111} \text {. }
\end{aligned}
$$

\subsection{Nonlinear Schrödinger hierarchy}

The nonlinear Schrödinger equation is one of the most prominent integrable PDEs $[11,12]$. The corresponding hierarchy is discussed for example in [1,21]. It is a special case of the AKNS hierarchy, the pluri-Lagrangian structure of which is studied in [24]. Here we construct a pluri-Lagrangian structure for the NLS hierarchy using Theorem 4.2.

In this example we consider a complex field $u: \mathbb{R}^{N} \rightarrow \mathbb{C}$. The first two equations of the hierarchy are the nonlinear Schrödinger equation itself and the complex modified $\mathrm{KdV}$ equation,

$$
\begin{aligned}
& u_{2}=i u_{11}-2 i|u|^{2} u, \\
& u_{3}=u_{111}-6|u|^{2} u_{1} .
\end{aligned}
$$

Fields $u$ that solve both these equations and their complex conjugates are critical fields for the Lagrangians (see e.g. [3])

$$
\begin{aligned}
L_{12}[u] & =\frac{i}{2}\left(u_{2} \bar{u}-u \bar{u}_{2}\right)-\left|u_{1}\right|^{2}-|u|^{4}, \\
L_{13}[u] & =\frac{i}{2}\left(u_{3} \bar{u}-u \bar{u}_{3}\right)+\frac{i}{2}\left(u_{11} \bar{u}_{1}-u_{1} \bar{u}_{11}\right)+\frac{3 i}{2}|u|^{2}\left(u_{1} \bar{u}-u \bar{u}_{1}\right) .
\end{aligned}
$$

For these Lagrangians Lemma 4.1 gives us the function

$$
\begin{aligned}
F_{23}\left(u, u_{1}, u_{11}, \ldots\right)=2|u|^{6} & -\frac{3}{2}|u|^{2}\left(u_{11} \bar{u}-u \bar{u}_{11}\right)-6\left|u u_{1}\right|^{2} \\
& +\frac{1}{2}\left(u_{111} \bar{u}_{1}+u_{1} \bar{u}_{111}\right)+\left|u_{11}\right|^{2}
\end{aligned}
$$

and Theorem 4.2 provides the coefficient

$$
\begin{aligned}
L_{23}[u]=-4|u|^{6}-u_{1}^{2} \bar{u}^{2} & -u^{2} \bar{u}_{1}^{2}+2\left|u u_{1}\right|^{2}+2|u|^{2}\left(u_{11} \bar{u}+u \bar{u}_{11}\right) \\
& +\frac{3}{2} i|u|^{2}\left(u_{2} \bar{u}-u \bar{u}_{2}\right)+\frac{i}{2}\left(u_{12} \bar{u}_{1}-u_{1} \bar{u}_{12}\right) \\
& +u_{3} \bar{u}_{1}+u_{1} \bar{u}_{3}-\left|u_{11}\right|^{2}+i\left(u_{11} \bar{u}_{2}-u_{2} \bar{u}_{11}\right)
\end{aligned}
$$

of a pluri-Lagrangian 2-form

$$
\mathcal{L}[u]=L_{12}[u] \mathrm{d} t_{1} \wedge \mathrm{d} t_{2}+L_{13}[u] \mathrm{d} t_{1} \wedge \mathrm{d} t_{3}+L_{23}[u] \mathrm{d} t_{2} \wedge \mathrm{d} t_{3} .
$$


Interestingly, in this example the classical Euler-Lagrange equations

$$
\frac{\delta_{12} L_{12}}{\delta u}=0 \text { and } \frac{\delta_{13} L_{13}}{\delta u}=0
$$

already yield the evolutionary form of the NLS equations (25)-(26). All other multitime Euler-Lagrange equations, in particular those of the form

$$
\frac{\delta_{12} L_{12}}{\delta u_{1}}=-\frac{\delta_{23} L_{23}}{\delta u_{3}} \text { and } \frac{\delta_{13} L_{13}}{\delta u_{1}}=\frac{\delta_{23} L_{23}}{\delta u_{2}}
$$

are trivially satisfied.

\subsection{Sine-Gordon equation and modified KdV hierarchy}

Consider the sine-Gordon equation

$$
u_{12}=\sin u
$$

and the (potential) modified KdV hierarchy

$$
\begin{aligned}
& u_{3}=u_{111}+\frac{1}{2} u_{1}^{3}, \\
& u_{4}=\frac{3}{8} u_{1}^{5}+\frac{5}{2} u_{1} u_{11}^{2}+\frac{5}{2} u_{1}^{2} u_{111}+u_{11111},
\end{aligned}
$$

This hierarchy consists of symmetries of the sine-Gordon equation (see, e.g. [19] or [17, Section 5k]). The corresponding Lagrangians are

$$
\begin{aligned}
& L_{12}[u]=\frac{1}{2} u_{1} u_{2}-\cos u, \\
& L_{13}[u]=\frac{1}{2} u_{1} u_{3}-\frac{1}{8} u_{1}^{4}+\frac{1}{2} u_{11}^{2}, \\
& L_{14}[u]=\frac{1}{2} u_{1} u_{4}-\frac{1}{16} u_{1}^{6}-\frac{5}{12} u_{1}^{3} u_{111}-\frac{1}{2} u_{111}^{2},
\end{aligned}
$$

Since the sine-Gordon equation is not evolutionary, Theorem 4.2 does not apply to this hierarchy. Surprisingly, a naive adaptation of the construction leads to a suitable 2-form, at least for the first few equations of the hierarchy.

We start the construction of a pluri-Lagrangian 2-form in three dimensions, considering only $t_{1}, t_{2}$ and $t_{3}$. Let $Q_{3}=u_{111}+\frac{1}{2} u_{1}^{3}$. Then on solutions of the equations, there holds 


$$
\begin{aligned}
\mathrm{D}_{2} L_{13}-\mathrm{D}_{3} L_{12} & =\frac{1}{2} u_{12} u_{3}-\frac{1}{2} u_{1}^{3} u_{12}+u_{11} u_{112}-\frac{1}{2} u_{13} u_{2}-u_{3} \sin u \\
& =-\frac{1}{2} u_{12} Q_{3}-\frac{1}{2} u_{2} \mathrm{D}_{1} Q_{3}-\frac{1}{2} u_{1}^{3} \sin u+u_{11} u_{1} \cos u \\
& =\mathrm{D}_{1} F_{23}
\end{aligned}
$$

for

$$
F_{23}[u]=-\frac{1}{2} u_{2}\left(u_{111}+\frac{1}{2} u_{1}^{3}\right)+\frac{1}{2} u_{1}^{2} \cos u .
$$

Since there is no evolutionary equation for $u_{2}$, we tolerate the dependence of $F_{23}$ on this derivative. For the same reason, the term $\frac{\delta_{12} L_{12}}{\delta u_{1} \alpha+1} \mathrm{D}_{1}^{\alpha}\left(u_{2}-Q_{2}\right)$ in equation (16) only makes sense for $\alpha>0$. For $\alpha=0$ we just remove it. We are left with

$$
\begin{aligned}
L_{23}[u]= & \frac{\delta_{13} L_{13}}{\delta u_{11}}\left(u_{12}-\sin u\right)-\frac{\delta_{12} L_{12}}{\delta u_{1}}\left(u_{3}-u_{111}-\frac{1}{2} u_{1}^{3}\right)+F_{23}[u] \\
= & u_{11}\left(u_{12}-\sin u\right)-\frac{1}{2} u_{2}\left(u_{3}-u_{111}-\frac{1}{2} u_{1}^{3}\right) \\
& -\frac{1}{2} u_{2}\left(u_{111}+\frac{1}{2} u_{1}^{3}\right)+\frac{1}{2} u_{1}^{2} \cos u \\
= & u_{11}\left(u_{12}-\sin u\right)-\frac{1}{2} u_{2} u_{3}+\frac{1}{2} u_{1}^{2} \cos u .
\end{aligned}
$$

This pluri-Lagrangian structure in $\mathbb{R}^{3}$ was first found in [27], but a pluri-Lagrangian structure incorporating more equations of the hierarchy has not been given previously. With the method presented here, such an extension is obtained by a straightforward (but long) calculation. For example, we can calculate $F_{24}$ and $F_{34}$ analogously to $F_{23}$ above. This in turn allow us to calculate the coefficients of the Lagrangian 2-form,

$$
\begin{gathered}
L_{24}[u]=\frac{3}{8} u_{1}^{4} \cos u-\frac{5}{12} u_{1}^{3} u_{112}+\frac{5}{4} u_{1}^{2} u_{11} u_{12}-\frac{3}{2} u_{1}^{2} u_{11} \sin u-\frac{1}{2} u_{11}^{2} \cos u \\
+u_{1} u_{111} \cos u-u_{111} u_{112}+u_{1111} u_{12}-\frac{1}{2} u_{2} u_{4}-u_{1111} \sin u
\end{gathered}
$$

and

$$
\begin{aligned}
L_{34}[u]= & \frac{3}{128} u_{1}^{8}-\frac{5}{16} u_{1}^{4} u_{11}^{2}+\frac{7}{16} u_{1}^{5} u_{111}-\frac{3}{16} u_{1}^{5} u_{3}-\frac{1}{8} u_{11}^{4}+\frac{7}{4} u_{1} u_{11}^{2} u_{111} \\
& +\frac{3}{4} u_{1}^{2} u_{111}^{2}-\frac{3}{2} u_{1}^{2} u_{11} u_{1111}+\frac{1}{4} u_{1}^{3} u_{11111}-\frac{5}{12} u_{1}^{3} u_{113}+\frac{5}{4} u_{1}^{2} u_{11} u_{13} \\
& -\frac{5}{4} u_{1} u_{11}^{2} u_{3}-\frac{5}{4} u_{1}^{2} u_{111} u_{3}+\frac{1}{4} u_{1}^{3} u_{4}-\frac{1}{2} u_{1111}^{2}+\frac{1}{2} u_{111} u_{11111} \\
& -u_{111} u_{113}+u_{1111} u_{13}-u_{11} u_{14}-\frac{1}{2} u_{11111} u_{3}+\frac{1}{2} u_{111} u_{4} .
\end{aligned}
$$


The presented hierarchy can be extended to a doubly-infinite hierarchy, where the sine-Gordon equation connects two copies of the modified KdV hierarchy, one as stated above and one where $t_{2}$ is used as space variable. The calculations presented here can be easily extended to cover both sides of the hierarchy. A pluri-Lagrangian structure of this double hierarchy was previously obtained using a carefully chosen continuum limit [29].

In this example, a straightforward adaptation of equation (16) gives us suitable coefficients $L_{i j}$. However, there does not seem to be a simple generalization of the proof we gave for Theorem 4.2 to cover this case. In this example we have verified by direct calculation that the multi-time Euler-Lagrange equations consist of the SineGordon and modified KdV equations and differential consequences thereof. Showing the validity of our construction in a more general setting, ideally with a more conceptual proof, is a goal for future research.

\section{Conclusions}

We have shown that a hierarchy of 2-dimensional variational PDEs, that are variational symmetries of each other, possesses a pluri-Lagrangian structure. This extends the results of [22], where a similar result was obtained for variational ODEs. The existence of a hierarchy of variational symmetries for a PDE is closely related to its integrability. Hence our result contributes significantly to the evidence that pluriLagrangian structures are a fundamental feature of integrability. Furthermore, our construction can be used to obtain new examples of pluri-Lagrangian 2-forms, as we illustrated in the context of the nonlinear Schrödinger hierarchy.

As illustrated by the example of the sine-Gordon and $\mathrm{mKdV}$ equations, our construction applies more generally than the proof we provided. More research is needed to determine the most general form of the ideas presented here. Relevant to this line of investigation is the paper [25], which deals with the same topics as the present work (and appeared on the arXiv one day after it).

Acknowledgements The authors are grateful to Yuri Suris for helpful discussions and feedback on a draft of this manuscript.

Funding Open Access funding enabled and organized by Projekt DEAL.

Open Access This article is licensed under a Creative Commons Attribution 4.0 International License, which permits use, sharing, adaptation, distribution and reproduction in any medium or format, as long as you give appropriate credit to the original author(s) and the source, provide a link to the Creative Commons licence, and indicate if changes were made. The images or other third party material in this article are included in the article's Creative Commons licence, unless indicated otherwise in a credit line to the material. If material is not included in the article's Creative Commons licence and your intended use is not permitted by statutory regulation or exceeds the permitted use, you will need to obtain permission directly from the copyright holder. To view a copy of this licence, visit http://creativecommons.org/licenses/by/4.0/. 


\section{References}

1. Anco, S.C., Mobasheramini, F.: Integrable $U(1)$-invariant peakon equations from the NLS hierarchy. Phys. D 355, 1-23 (2017)

2. Atkinson, J., Lobb, S.B., Nijhoff, F.W.: An integrable multicomponent quad-equation and its Lagrangian formulation. Theor. Math. Phys. 173(3), 1644-1653 (2012)

3. Avan, J., Caudrelier, V., Doikou, A., Kundu, A.: Lagrangian and Hamiltonian structures in an integrable hierarchy and space-time duality. Nuclear Phys. B 902, 415-439 (2016)

4. Bobenko, A.I., Suris, Yu.B.: Discrete pluriharmonic functions as solutions of linear pluri-Lagrangian systems. Commun. Math. Phys. 336(1), 199-215 (2015)

5. Boll, R., Petrera, M., Suris, Yu.B.: Multi-time Lagrangian 1-forms for families of Bäcklund transformations. Toda-type systems. J. Phys. A 46(27), \# 275204 (2013)

6. Boll, R., Petrera, M., Suris, Yu.B.: What is integrability of discrete variational systems? Proc. R. Soc. London Ser. A Math. Phys. Eng. Sci. 470(2162), \# 20130550 (2014)

7. Boll, R., Petrera, M., Suris, Yu.B.: Multi-time Lagrangian 1-forms for families of Bäcklund transformations. Relativistic Toda-type systems. J. Phys. A 48(8), \# 085203 (2015)

8. Boll, R., Petrera, M., Suris, Yu.B.: On the variational interpretation of the discrete KP equation. In: Bobenko, A.I. (ed.) Advances in Discrete Differential Geometry, pp. 379-405. Springer, Berlin (2016)

9. Boll, R., Petrera, M., Suris, Yu.B.: On integrability of discrete variational systems: octahedron relations. Int. Math. Res. Not. IMRN 2016(3), 645-668 (2016)

10. Dickey, L.A.: Soliton Equations and Hamiltonian Systems. Advanced Series in Mathematical Physics, vol. 12. World Scientific, Singapore (1991)

11. Faddeev, L.D., Takhtajan, L.A.: Hamiltonian Methods in the Theory of Solitons. Classics in Mathematics. Translated from Russian by A.G. Reyman. Springer, Berlin (2007)

12. Kaup, D.J., Newell, A.C.: An exact solution for a derivative nonlinear Schrödinger equation. J. Math. Phys. 19(4), 798-801 (1978)

13. Lobb, S., Nijhoff, F.: Lagrangian multiforms and multidimensional consistency. J. Phys. A 42(45), \# 454013 (2009)

14. Lobb, S., Nijhoff, F.W.: Lagrangian multiform structure for the lattice Gel'fand-Dikij hierarchy. J. Phys. A 43(7), \# 072003 (2010)

15. Lobb, S.B., Nijhoff, F.W.: A variational principle for discrete integrable systems. SIGMA Symmetry Integrability Geom. Methods Appl. 14, \# 041 (2018)

16. Lobb, S.B., Nijhoff, F.W., Quispel, G.R.W.: Lagrangian multiform structure for the lattice KP system. J. Phys. A 42(47), \# 472002 (2009)

17. Newell, A.C.: Solitons in Mathematics and Physics. CBMS-NSF Regional Conference Series in Applied Mathematics, vol. 48. Society for Industrial and Applied Mathematics, Philadelphia (1985)

18. Noether, E.: Invariante Variationsprobleme. Nachr. Ges. Wiss. Göttingen Math.-Phys. K1. 1918, 235257 (1918)

19. Olver, P.J.: Evolution equations possessing infinitely many symmetries. J. Math. Phys. 18(6), 12121215 (1977)

20. Olver, P.J.: Applications of Lie Groups to Differential Equations. 2nd ed. Graduate Texts in Mathematics, vol. 107. Springer, New York (1993)

21. Olver, P.J., Rosenau, P.: Tri-Hamiltonian duality between solitons and solitary-wave solutions having compact support. Phys. Rev. E 53(2), 1900-1906 (1996)

22. Petrera, M., Suris, Yu.B.: Variational symmetries and pluri-Lagrangian systems in classical mechanics. J. Nonlinear Math. Phys. 24(suppl. 1), 121-145 (2017)

23. SageMath.: the Sage Mathematics Software System (Version 8.1). https://www.sagemath.org (2017)

24. Sleigh, D., Nijhoff, F., Caudrelier, V.: A variational approach to Lax representations. J. Geom. Phys. 142, 66-79 (2019)

25. Sleigh, D., Nijhoff, F., Caudrelier, V.: Variational symmetries and Lagrangian multiforms. Lett. Math. Phys. 110(4), 805-826 (2020)

26. Suris, Yu.B.: Variational formulation of commuting Hamiltonian flows: multi-time Lagrangian 1-forms. J. Geom. Mech. 5(3), 365-379 (2013)

27. Suris, Yu.B.: Variational symmetries and pluri-Lagrangian systems. In: Hagen, T., et al. (eds.) Dynamical Systems, Number Theory and Applications, pp. 255-266. A Festschrift in honor of Armin Leutbecher's 80th birthday. World Scientific, Hackensack (2016) 
28. Suris, Yu.B., Vermeeren, M.: On the Lagrangian structure of integrable hierarchies. In: Bobenko, A.I. (ed.) Advances in Discrete Differential Geometry, pp. 347-378. Springer, Berlin (2016)

29. Vermeeren, M.: A variational perspective on continuum limits of ABS and lattice GD equations. SIGMA Symmetry Integrability Geom. Methods Appl. 15, \# 044 (2019)

30. Vermeeren, M.: Continuum limits of pluri-Lagrangian systems. J. Integrable Syst. 4(1), \# 020 (2019)

31. Vermeeren, M.: Support code for "Variational symmetries and pluri-Lagrangian structures for integrable hierarchies of PDEs". https://doi.org/10.5281/zenodo.3243313

32. Xenitidis, P., Lobb, S., Nijhoff, F.: On the Lagrangian formulation of multidimensionally consistent systems. Proc. R. Soc. London Ser. A Math. Phys. Eng. Sci. 467(2135), 3295-3317 (2011)

33. Yoo-Kong, S., Lobb, S., Nijhoff, F.: Discrete-time Calogero-Moser system and Lagrangian 1-form structure. J. Phys. A 44(36), \# 365203 (2011)

Publisher's Note Springer Nature remains neutral with regard to jurisdictional claims in published maps and institutional affiliations. 